CP, 2018, Vol.7 - No13, pp. 9/26 ISSN 2014-6752. Girona (Catalunya). Universitat de Girona. TERRÓN BLANCO, J.L. ; RAMÍREZ LEYVA, J.M.; VIALÁS FERNÁNDEZ, S.; JACOBETTY P.: La interdisciplinariedad en los artículos de comunicación de las revistas de salud pública de SCIELO Recibido: 01/12/2017 - Aceptado: 13/2/2018

\title{
La interdisciplinariedad en los artículos de comunicación de las revistas de salud pública de SCIELO
}

\section{Interdisciplinarity in the communication articles of SCIELO's public health journals}

\author{
Autores \\ José Luis Terrón Blanco \\ InCom-UAB, joseluis.terron@uab.cat \\ Flor Micaela Ramírez Leyva \\ Universidad de Guadalajara, México, lor.ramirez@cuc.udg.mx \\ Simón Vialás Fernández \\ InCom-UAB, simovf@gmail.com \\ Pedro Jacobetty \\ UOC, pedro.jacobetty@gmail.com
}

\section{Resumen:}

La comunicación debería de ser un campo esencial para la salud pública (SP), dado que sus actuaciones, a menudo, son actos comunicativos o intervenciones que persiguen la promoción de la salud. Por otro lado, todos los autores estudiados sostienen que una de las características de la SP es un interdisciplinariedad. En esta investigación nos preguntamos hasta qué punto las publicaciones de SP insertan artículos en los que la comunicación es protagónica y si esos artículos son interdisciplinares. Se trata de una investigación exploratoria, cuya metodología es el análisis de contenido; para llevarla a cabo se realizó una revisión automática y manual de los textos que se han sometido al estudio. La muestra está compuesta por todos los a artículos de las revistas de salud pública (18) de la plataforma

\section{Abstract:}

Communication should be an essential field for public health, as its actions are often communicative acts or interventions aimed at health promotion. On the other hand all the authors studied maintain that one of the characteristics of the public health is interdisciplinarity. In this research we asked ourselves to what extent the public health publications insert articles in which communication plays a leading role and whether these articles are interdisciplinary. This is an exploratory investigation, the methodology of which is content analysis; to carry it out, an automatic and manual revision of the texts that have been submitted to the study was carried out. The sample is composed of all articles from the public health journals (18) of the SCIELO platform between 2005 and 2015 (included). Of the total number 
SCIELO entre los años 2005 y 2015 (incluidos). Del total de artículos (19.886) se escogieron aquellos que la propia plataforma caracteriza como de 'comunicación' $(n=124)$, y de estos, los que eran interdisciplinares $(n=23)$. Entre los resultados cabe destacar el bajo grado de interdisciplinariedad, que esta se manifiesta, excepto en 3 casos, de manera binaria, siendo la relación más prevalente la de los campos de la salud y de las ciencias sociales y jurídicas; sólo en 6 artículos hay interdisciplinariedad entre los campos de la salud y de la comunicación. También hemos de destacar que la mayoría de los IP (52\%) y colaboradores (48\%) son del campo de la salud y que la interdisciplinariedad aparece solo en 9 revistas, dándose una concentración de artículos en 1, Interface, con 11.

Palabras clave: salud pública:

comunicación; interdisciplinariedad; revistas; artículos. of articles $(19,886)$, those chosen were those that the platform itself characterizes as' communication' $(n=124)$, and of these, those that were interdisciplinary $(n=23)$. Among the results, it is worth highlighting the low degree of interdisciplinarity, which is manifested, except in 3 cases, in a binary way, with the most prevalent relationship being that of the health, social and legal sciences; only in 6 articles there is interdisciplinarity between the fields of health and communication. It should also be noted that the majority of senior researchers (52\%) and collaborators $(48 \%)$ are in the health field and that interdisciplinarity appears only in 9 journals, with a concentration of articles in 1 , Interface, with 11.

Key words: public health: communication; interdisciplinarity; journals; articles.

\section{Introducción}

Existe un amplio consenso en el carácter interdisciplinario de la Salud Pública (de Souza, 2008; Baum, 2007; Ramos Domínguez, 2000; Vega Franco, 2000; Almeida Filho y Paim, 1999), que, como asegura Franco (2006: 119) es un "campo de conocimiento en trasformación transdisciplinar", por lo que, consecuentemente, ha ido variando en su concepción a partir de los contextos sociopolíticos que, como en el caso de otras disciplinas, han incidido en la continua reconstrucción, ampliación y evolución de este campo (Kottow, 2013; Vega Franco, 2000; Navarro, 1998; Frenk, 1992; Testa, 1992).

Las definiciones clásicas de la SP de Winslow (de 1920) y Piédrola Gil (de 1991) -Véase Barragán (2007)- comparten su carácter de ciencia y arte para orientar el esfuerzo organizado comunitario con la finalidad de impedir enfermedades y proteger y fomentar la salud, por lo que enfatizan la responsabilidad colectiva de la población. Frenk (1992: 12) la entiende como "la aplicación de las ciencias sociales, biológicas y de conducta, al estudio de las poblaciones humanas con 2 objetivos: el estudio epidemiológico de las condiciones de salud y la investigación de los servicios de salud. Su esencia es la Salud de la Población", en tanto que Ramos Domínguez (2000: 83) ve a la SP como multidisciplinaria y de amplio alcance; su enfoque es individual y colectivo (poblacional); "la salud y la enfermedad se consideran como un proceso determinado por múltiples causas" (lo que supone tener en cuenta a los determinantes sociales), tiene una orientación integral y su finalidades primordiales serían la promoción de la salud y la prevención de las enfermedades.

La OPS (2012: 14), por su parte, la entiende como una "práctica social integrada que tiene 
como sujeto y objeto de estudio, la salud de las poblaciones humanas y se le considera como la ciencia encargada de prevenir la enfermedad, la discapacidad, prolongar la vida, fomentar la salud física y mental, mediante los esfuerzos organizados de la comunidad, para el saneamiento del ambiente y desarrollo de la maquinaria social, para afrontar los problemas de salud y mantener un nivel de vida adecuado".

El reconocimiento de la interdisciplinariedad de la SP se puso de relieve desde 1974 con el Informe Lalonde, en el cual se delimitaron cuatro nodos del modelo de "campo de la salud": la biología humana, el sistema organizado de servicios (de recuperación, curativo y preventivo); el ambiente, (lo social, lo psicológico y lo físico); y el estilo de vida. Iniciativas posteriores de la OPS promovieron el desarrollo de diversas áreas críticas, tales como las políticas públicas, los sistemas de información y desarrollo de la epidemiología, economía y financiamiento, recursos humanos en salud, desarrollo tecnológico y sistemas de servicios de salud (Almeida Filho y Paim, 1999).

Según la OPS (2012), las funciones de la salud pública serían las de monitoreo de la situación de salud, la vigilancia epidemiológica, la promoción de la salud y el empoderamiento de la gente, la participación social y la intersectorialidad, la planificación estratégica en salud pública, la regulación y fiscalización, la garantía de acceso a la atención de salud, la evaluación de eficacia, acceso y calidad de los servicios de salud, la reducción del impacto de emergencia y desastres en salud y la investigación, desarrollo e implementación de soluciones innovadoras. Por todo ello considerará que les son propias las siguientes actividades: la protección de la salud, la promoción de la salud, la prevención de la enfermedad y la restauración de la salud.

Revuelta, Faubel y Carrasco (2014: 8), sostienen que si existe "un ámbito de la salud en que la comunicación es absolutamente esencial es en el de la salud pública (SP), ya que sus mismas intervenciones a menudo son puros actos comunicativos, con la intención de modificar comportamiento o actitudes"; estos autores (2014: 8) añaden que es "importante conocer cuáles son las características fundamentales de la comunicación en SP, qué papel tienen los distintos actores que intervienen y cuáles son las líneas que se perfilan en un futuro a corto y medio plazo".

La importancia e incidencia de la cuestión interdisciplinar entre comunicación y salud ha sido reiteradamente vindicada (Lois, 2015; Petracci y Waisbord, 2011; Scliar, 2002); no obstante, se ha discutido si el rol de la comunicación para la SP -como para el campo de la salud en general- se reduce a una función instrumental (Lois, 2015; Petracci y Waisbord, 2011) y no existe, por tanto, la necesaria interdisciplinariedad entre ambos campos. La propia OPS (2012: 25) le concede gran importancia a la información/comunicación y a la cultura, pero entre las disciplinas que integran la SP no incluye la comunicación, aunque si otras del campo de las ciencias sociales: economía, sociología, ciencias políticas, antropología y demografía. Por omisión, desde nuestro punto de vista, subraya el carácter meramente instrumental de la comunicación.

\section{Metodología}

El trabajo que ahora presentamos forma parte de una investigación más amplia cuya intención es caracterizar, grosso modo, los artículos sobre comunicación publicados en las revistas de 
salud pública, intentando saber su cantidad, quiénes son sus emisores, de qué tratan y qué características tienen en común.

Para ello, se ha conformado un objeto de estudio con todas las revistas de salud pública de la plataforma SCIELO. Hemos escogido esta biblioteca digital por incluirse publicaciones de libre acceso, porque pertenecen a distintos países de Latinoamérica, y porque forman parte de una plataforma con unos estándares de calidad reconocidos, a la vez que es muy consultada.

La muestra se analizó entre los años 2005 y 2015 (ambos incluidos). El universo a tener en cuenta es de 18 publicaciones, que se publican en 11 países distintos: Annali dell'Istituto Superior de Sanità (Italia), Bulletin of the World Health Organization (Suiza), Cadernos de Saúde Pública (Brasil), Ciência \& Saúde Coletiva (Brasil), Epidemiologia e Serviços de Saúde (Brasil), Gaceta Sanitaria (España), Interface - Comunicação, Saúde, Educação (Brasil), MEDICC Review (Cuba), Physis: Revista de Saúde Coletiva (Brasil), Revista Brasileira de Epidemiologia (Brasil), Revista Cubana de Salud Pública (Cuba), Revista Española de Salud Pública (España), Revista Panamericana de Salud Pública (EUA), Revista Peruana de Medicina Experimental y Salud Pública (Perú), Revista de Salud Pública (Colombia), Revista de Saúde Pública (Brasil), Salud Colectiva (Argentina), Salud Pública de México (México). SCIELO incluye a las 18 revistas en el ámbito de ciencias de la salud, excepto a dos (Interface - Comunicação, Saúde, Educação y Physis: Revista de Saúde Coletiva ), que las circunscribe a su vez en el ámbito de las humanidades. Ahora bien, de estas 18 revistas 6 han sido excluidas de nuestro estudio dado que según el buscador de SCIELO no insertan, en el periodo analizado, ningún artículo que se centre en comunicación. Las publicaciones excluidas fueron las siguientes: Annali dell'Istituto Superiore di Sanità (Italia), Bulletin of the World Health Organization (Suiza) -ausencia que nos aprece relevante-, MEDICC Review (Cuba), Epidemiologia e Serviços de Saúde (Brasil), Physis: Revista de Saúde Coletiva (Brasil) y Revista Brasileira de Epidemiologia (Brasil).

El total de artículos a considerar, por tanto, fue de 19.886, de los cuales analizamos 124, aquellos que según el propio buscador de SCIELO tienen como uno de sus argumentos centrales la comunicación.

Para llevar a cabo nuestra investigación hacemos uso del análisis de contenido y del bibliométrico. A la hora de realizar el análisis de contenido hemos prestado nuestra atención en los titulares, resúmenes y palabras claves, salvo en los artículos en los que no se especificaba el enfoque metodológico en el resumen, que fueron leídos en su integridad para intentar averiguar si aparecían en su cuerpo. El análisis de contenido se realizó de maneras automática y manual, creando tablas excel; la descarga automatizada de los atributos de cada artículo se hizo a través de scraping, con recurso al lenguaje de programación Python, y la producción de tablas con LibreOffice.

Manualmente hemos revisado y clasificado a los autores (tanto al investigador principal -IPcomo a sus colaboradores) en relación a 5 campos del conocimiento, clasificación empleada por muchas universidades españolas: artes y humanidades; ciencias de la naturaleza; ciencias sociales y jurídicas; ciencias de la salud. A estos 5 campos le hemos añadido uno más, comunicación, que forma parte de las ciencias sociales y jurídicas, pero que a tenor del carácter de esta investigación nos parece oportuno segregar. Los autores se adscribieron a un 
campo de conocimiento a partir de las filiaciones que ofrecían las revistas de los mismos en los originales (Terrón, Ramírez, Vialás y Jacobetty, 2016).

Como hemos escrito, se ha organizado la información en un cuadro de Excel, en el que se ha tenido en cuenta, de cada artículo, su título, año, revista, nombre de sus autores, campo de conocimiento y filiaciones, centrándonos, principalmente, en los IP y buscando posibles relaciones entre los mimos o entre sus colaboradores o instituciones de pertenencia. Por otra parte, los contenidos de los artículos los hemos obtenido agrupando las palabras claves de los mismos, dada la gran dispersión que hemos encontrado.

Para analizar los enfoques metodológicos nos hemos basado en las investigaciones de Martínez Nicolás y Saperas Lapiedra $(2011,2016)$. Siguiendo su metodología, hemos considerado 5 tipos de investigación: teoría y conceptualización, metodología, revisión, empíricos y sin especificar. A estos últimos, Martínez Nicolás y Saperas Lapiedra (2016: 1638) los denominan "investigación empírica sin técnicas sistemáticas", y como nos detallan "en esta categoría fueron clasificados los trabajos metodológicamente deficientes; es decir, aquellos que, abordando aspectos fenoménicos de la comunicación (es decir, la propia realidad empírica), no apoyan los resultados que obtienen en datos obtenidos mediante procedimientos estandarizados (técnicas sistemáticas o análisis de fuentes documentales), hallándose más cerca del comentario, la reflexión personal o la descripción ocasional o intuitiva que del rigor exigible a la investigación científica".

Los empíricos los subdividimos en tres categorías que, a su vez, incluyen los procedimientos sistemáticos o estandarizados en el campo de la salud pública: cuantitativos (análisis de contenido, encuestas, análisis secundarios de datos, cuestionarios, estudios de cohorte, test), cualitativos (entrevistas, grupos focales, análisis del discurso, observación participante y grupos de discusión) y mixtos (cuantitativos y cualitativos a la par).

Para establecer sus características formales hemos seguido la convención establecida por las revistas científicas de más impacto que distinguen entre 7 posibilidades: artículo original, reseña bibliográfica, carta al editor, comentario, editorial, revisión y perspectivas.

El propósito de la investigación que presentamos en este escrito intenta responder a las siguientes preguntas:

1. ¿Cuántos artículos centrados en comunicación aparecen en las revistas de salud pública estudiadas?

2. ¿Cuáles son sus características formales?

3. ¿Hay interdisciplinariedad?

4. ¿Se trabaja interdisciplinarmente?

5. ¿Cuántos artículos están firmados por equipos interdisciplinarios?

6. ¿Cuáles son los campos de conocimiento de los autores de los artículos?

7. ¿De qué tratan?

8. ¿Cuáles son sus enfoques metodológicos?

9. ¿Qué diferencias y similitudes hay en las revistas estudiadas?

Mientras que la primera cuestión ( $y$, en parte, la novena) se refieren a la muestra de los 124 
artículos que según la propia biblioteca SCIELO se centran en la comunicación, el resto de las preguntas, que de una u otra forma ponen su foco de atención en la interdisciplinariedad, servirán para analizar los 23 artículos interdisciplinares que hemos encontrado entre los 124 artículos antes referidos.

\section{Resultados}

En primer lugar, hemos de recordar que de las 18 revistas de salud pública integradas en la biblioteca digital de SCIELO, sólo 12 publicaciones insertaban en el periodo estudiado -2005 y 2015 (ambos incluidos)-, artículos que se centraban en la comunicación. Las 12 revistas estudiadas publicaban 19.866 artículos, pero únicamente 124, apenas un 0,63\%, del total, tenían como argumento central a la comunicación. Esos 124 escritos conforman, en principio, nuestra muestra.

Tabla 1: Número de artículos que se centran en comunicación en relación con el total de artículos. Por años y total.

\begin{tabular}{|l|l|l|l|}
\hline AÑO & $\begin{array}{l}\text { TOTAL } \\
\text { ARTÍ́CULOS }\end{array}$ & ARTíCULOS COM. & $\%$ \\
\hline 2005 & 1392 & 9 & 0,646 \\
\hline 2006 & 1600 & 10 & 0,625 \\
\hline 2007 & 1827 & 4 & 0,218 \\
\hline 2008 & 1986 & 7 & 0,352 \\
\hline 2009 & 2009 & 16 & 0,796 \\
\hline 2010 & 2069 & 6 & 0,289 \\
\hline 2011 & 2308 & 17 & 0,736 \\
\hline 2012 & 2077 & 12 & 0,577 \\
\hline 2013 & 2234 & 8 & 0,358 \\
\hline 2014 & 2230 & 17 & 0.762 \\
\hline 2015 & 2231 & 13 & 0,582 \\
\hline TOTAL & 19886 & 124 & 0,629 \\
\hline
\end{tabular}

Fuente: elaboración propia

El número de artículos varía, de menos a más, entre los 4 de 2007 y los 17 de 2011. Si observamos detenidamente la tabla nos daremos cuenta que no es posible establecer ninguna tendencia creciente ni decreciente.

Por su parte, en la Tabla 2 se indica el número artículos que conforman la muestra atendiendo a la revista que los incluyen. 


\section{Tabla 2. Número de artículos por revistas}

\begin{tabular}{|l|l|}
\hline Revista & Sum - n \\
\hline Cadernos de Saúde Pública & 3 \\
\hline Ciência \& Saúde Coletiva & 2 \\
\hline Gaceta Sanitaria & 10 \\
\hline Interface - Comunicação, Saúde, Educação & 51 \\
\hline Revista Cubana de Salud Pública & 5 \\
\hline Revista de Salud Pública & 10 \\
\hline Revista de Saúde Pública & 3 \\
\hline Revista Española de Salud Pública & 2 \\
\hline Revista Panamericana de Salud Pública & 14 \\
\hline Revista Peruana de Medicina Experimental y Salud Pública & 4 \\
\hline Salud Colectiva & 8 \\
\hline Salud Pública de México & 12 \\
\hline Total & 124 \\
\hline
\end{tabular}

Fuente: elaboración propia

Como podemos apreciar, la mayoría de los artículos se concentran en pocas publicaciones, y de estas, sobre todo en Interface ( $41,12 \%$ de los artículos). Si tomamos en consideración las 5 revistas que insertan 10 o más artículos, su suma se corresponde con el 78,22\% del total. La media por revista (teniendo en cuenta que sólo aparecen en 88 ejemplares distintos) es de 10,33, pero la mediana desciende a 6,5 y su moda es 2 . También hemos de considerar que 55 artículos están escritos en español, 39 en portugués, 30 en inglés y ninguno en italiano (lenguas oficiales de las revistas estudiadas).

Por otro lado, sólo un ejemplar inserta 6 artículos originales. Se trata del vol.35, no.5-6, de Revista Panamericana de Salud Pública, editado en 2014 y dedicado al eHealth, el único que incluye un dossier sobre comunicación y tecnologías de la información. Una revista inserta 5 artículos (Interface, vol 15, n. 37; número dedicado a la salud colectiva) y dos revistas 4 artículos sobre comunicación (Salud Pública de México, vol.52 n.2 suplemento 2 y Salud Pública de México, vol.54 n.3; ambos números dedicados al tabaquismo). Del resto de los ejemplares, 20 insertan 2 artículos y 62, o sea, la mayoría, un sólo artículo.

Ahora bien, de fijarnos en cómo se distribuyen los artículos en el tiempo debemos destacar que sólo Interface incluye artículos en los 11 años analizados y sólo tres revistas lo hacen en 6 años o más (Revista Panamericana de Salud Pública -en 8 años distintos-, Gaceta Sanitaria -en 7- y Salud Colectiva -en 6-). A la vez, hemos de señalar que las revistas se editan en 8 países diferentes y que la mayoría de los artículos se han publicado en Brasil (59); pero de las 4 revistas brasileñas, recordemos que en Interface se publicaron 51 , o sea, que si descartásemos a Interface las diferencias entre países por el número de artículos no es significativa.

Pero de esos 124 escritos sólo 23 son interdisciplinarios, con otras palabras, sólo 23 cuentan con autores de campos de estudio diferentes, lo que supone un $18,5 \%$ sobre los 124 que tratan 
sobre comunicación, y un 0,11\% sobre el total de los artículos publicados por el conjunto de las revistas estudiadas. En este escrito nos fijaremos especialmente en estos 23 artículos.

Tabla 3. Artículos interdisciplinares

\begin{tabular}{|c|c|c|}
\hline Título artículo & Revista & Año \\
\hline $\begin{array}{l}\text { Impacto de advertencias sanitarias del empaquetado } \\
\text { de cigarros: un análisis comparativo en Brasil, } \\
\text { Uruguay y México }\end{array}$ & Salud Pública Méx. & 2010 \\
\hline $\begin{array}{l}\text { Valores percibidos en la publicidad de alimentos por } \\
\text { jóvenes con y sin trastornos de la conducta alimentaria }\end{array}$ & Salud Colectiva & 2015 \\
\hline $\begin{array}{l}\text { Homicidios diarios. Análisis del discurso periodístico } \\
\text { sobre homicidios por armas de fuego. Buenos Aires } \\
\text { (Argentina) 2001-2002 }\end{array}$ & Salud Colectiva & 2010 \\
\hline $\begin{array}{l}\text { Desarrollo de un servicio en línea para la gestión } \\
\text { tecnológica en salud }\end{array}$ & $\begin{array}{l}\text { Rev. Per. de Medici. } \\
\text { Exp. y Salud Púb. }\end{array}$ & 2015 \\
\hline $\begin{array}{l}\text { Survey of rubella knowledge and acceptability of } \\
\text { rubella vaccination among Brazilian adults prior to } \\
\text { mass vaccination }\end{array}$ & $\begin{array}{l}\text { Rev. Panam Salud } \\
\text { Pública }\end{array}$ & 2011 \\
\hline $\begin{array}{l}\text { Applying the mental models framework to carbon } \\
\text { monoxide risk in northern Mexico }\end{array}$ & $\begin{array}{l}\text { Rev. Panam Salud } \\
\text { Pública }\end{array}$ & 2009 \\
\hline $\begin{array}{l}\text { Inequidades en salud comunicativa: el caso del } \\
\text { aprendizaje inicial de la escritura }\end{array}$ & Rev. Salud Pública & 2012 \\
\hline $\begin{array}{l}\text { Evaluación de la estrategia de comunicación "Mejor } \\
\text { Hablemos" para promover la convivencia pacífica en } \\
\text { Cali, 1996-2000 }\end{array}$ & Rev. Salud Pública & 2006 \\
\hline $\begin{array}{l}\text { Que no tengan miedo de nosotros: el Chagas según } \\
\text { los propios protagonistas }\end{array}$ & Interface & 2015 \\
\hline $\begin{array}{l}\text { Una estrategia didáctica para la formación de } \\
\text { educadores de salud en Brasil: la indagación dialógica } \\
\text { problematizadora }\end{array}$ & Interface & 2014 \\
\hline $\begin{array}{l}\text { O Programa Saúde da Família no bairro do Bom } \\
\text { Retiro, SP, Brasil: a comunicação entre bolivianos e } \\
\text { trabalhadores de saúde }\end{array}$ & Interface & 2014 \\
\hline As representações da saúde bucal na mídia impressa & Interface & 2012 \\
\hline $\begin{array}{l}\text { Letramento funcional em saúde: reflexões e conceitos } \\
\text { sobre seu impacto na interação entre usuários, } \\
\text { profissionais e sistema de saúde }\end{array}$ & Interface & 2012 \\
\hline $\begin{array}{l}\text { Hanseníase e práticas da comunicação: estudo de } \\
\text { recepção de materiais educativos em um serviço de } \\
\text { saúde no Rio de Janeiro }\end{array}$ & Interface & 2012 \\
\hline
\end{tabular}




\begin{tabular}{|l|l|l|}
\hline $\begin{array}{l}\text { A prática de alfabetização em Informação e } \\
\text { Comunicação em Saúde: o olhar dos agentes } \\
\text { comunitários de Saúde sobre o projeto de Inclusão } \\
\text { Digital em Sergipe, Brasil }\end{array}$ & 2011 \\
\hline $\begin{array}{l}\text { Acervo de materiais educativos sobre hanseníase: um } \\
\text { dispositivo da memória e das práticas comunicativas }\end{array}$ & Interface & 2010 \\
\hline $\begin{array}{l}\text { Ciência e consenso: os diferentes tempos que } \\
\text { envolvem a pesquisa científica, a decisão política e a } \\
\text { opinião pública }\end{array}$ & Interface & 2009 \\
\hline $\begin{array}{l}\text { Uma contribuição da semiótica para a comunicação } \\
\text { visual na área da saúde }\end{array}$ & Interface & 2009 \\
\hline $\begin{array}{l}\text { A escuta sensível: um estudo sobre o relacionamento } \\
\text { entre pessoas e ambientes voltados para a saúde }\end{array}$ & Interface & 2008 \\
\hline $\begin{array}{l}\text { Grupos sobre alimentación saludable en Facebook: } \\
\text { características y contenidos }\end{array}$ & Gaceta Sanitaria & 2005 \\
\hline $\begin{array}{l}\text { Historia reciente de la cobertura periodística de la } \\
\text { violencia contra las mujeres en el contexto español } \\
\text { (1997-2001) }\end{array}$ & Gaceta Sanitaria & 2011 \\
\hline $\begin{array}{l}\text { Diseño y aplicación de un Programa de Comunicación } \\
\text { de Riesgos para la salud ambiental infantil en un sitio } \\
\text { contaminado con plomo y arsénico }\end{array}$ & Ciênc. Saúde Coletiva & \\
\hline $\begin{array}{l}\text { Hacia un marco conceptual para repensar la } \\
\text { accesibilidad cultural }\end{array}$ & Cad. Saúde Publica & 2014 \\
\hline
\end{tabular}

Fuente: elaboración propia

La interdisciplinariedad se concentra en 9 revistas de las 12 estudiadas y de las 18 consideradas en primera instancia. Como puede apreciarse, Interface (Botucatu) es la revista que encabeza la lista, con 11 escritos (sobre 23), es decir, casi el $50 \%$ de los trabajos, por tanto podría considerársele la más interdisciplinaria. Tres publicaciones, a saber, Revista Panamericana de Salud Pública, Rev. Salud Pública, y Salud Colectiva cuentan con 2 artículos cada una. En las cinco revistas restantes, sólo encontramos un artículo. Y de fijarnos en la columna de los años, somos incapaces de entrever tendencia alguna dada la dispersión de resultados en una muestra tan reducida.

Si tomamos como referencia los 124 escritos, y teniendo en cuenta que el número de autores es lógicamente superior al número de artículos, (387), $302(78,03 \%)$ son del campo de la salud, 40 (10,33\%) de la comunicación, $22(5,68 \%)$ de las ciencias sociales, 11 (2,84\%) de las ingenierías, 7 (1,82\%) de las ciencias de la naturaleza y $5(, 3 \%)$ de las artes y humanidades El autor principal fue del campo de la salud en 93 ocasiones, en 15 del campo de la comunicación, en 7 de las ciencias sociales, y en 3 de las ingenierías, de las ciencias y de las humanidades y artes. De los 93 artículos del campo de la salud sólo 12 tenían un solo autor (el 12,9\%); de los 15 del campo de la comunicación, 6 estaban firmados por un solo autor (el 40\%). Ahora bien, si tenemos en cuenta cada uno de los artículos y deparamos en los campos de sus autores principales, obtendremos los siguientes resultados: 103 de la salud, 21 de la comunicación, 14 
de las ciencias sociales, 6 de las ingenierías y 4 de las ciencias y de las artes y humanidades (Terrón, Ramírez, Vialás y Jacobetty, 2016).

De fijarnos en los 23 artículos identificados con equipos de trabajo interdisciplinarios, el 52\% de los mismos se inscribe en el campo de la salud, el $17 \%$ en ciencias de la naturaleza, el $13 \%$ en el de comunicación, el $9 \%$ en ciencias sociales y jurídicas (al que deberíamos añadir ese $9 \%$ de comunicación) y el $4,3 \%$ a ingenierías y a artes y humanidades.

\section{Tabla 4. Campos de conocimiento y número de investigadores en ellos}

\begin{tabular}{|c|c|c|c|c|c|}
\hline Campo & IP & $\%$ & Cols. & $\%$ & Instituciones en las que se afilian los IP \\
\hline Salud & 12 & 52 & 41 & 48 & $\begin{array}{l}\text { 1. Inst. Nal. de S.P./Univ. of South Carolina } \\
\text { 2. Inst. de Saúde Coletiva/Univ. Federal da } \\
\text { Bahía (ISC-UFBa) 3. Oficina de las Naciones } \\
\text { Unidas de Servicios para Proyectos (UNOPS) } \\
\text { 4. Univ. Nal. de Colombia 5.Secretaria de } \\
\text { Salud Departamental del Valle del Cauca } 6 . \\
\text { Univ. de São Paulo 7. Escola Nal. de Saúde } \\
\text { Pública Sérgio Arouca, Fundação Oswaldo } \\
\text { Cruz (Ensp/Fiocruz) 8. Univ. Federal de } \\
\text { Viçosa; 9. Ensp/Fiocruz; 10. Instituto Oswaldo } \\
\text { Cruz (IOC/Fiocruz); 11. Colegio Oficial de } \\
\text { Médicos de Barcelona/Univ. Oberta de } \\
\text { Catalunya; 12. Univ. de Alicante }\end{array}$ \\
\hline Ciencias & 4 & 17 & 4 & 5 & $\begin{array}{l}\text { 1. Consejo Nacional de Investigaciones } \\
\text { Científicas y Tecnológicas - Univ. Nal. de } \\
\text { la Plata; 2. Univ. Nal. de Córdoba; 3. Univ. } \\
\text { Estadual do Ceará - UECE; 4. Univ. Autónoma } \\
\text { de San Luis Potosí }\end{array}$ \\
\hline Comunicación & 3 & 13 & 11 & 13 & $\begin{array}{l}\text { 1. Ministério da Saúde; 2. Univ. Pompeu } \\
\text { Fabra; 3. Centro Universitário Metodista } \\
\text { Izabela Hendrix }\end{array}$ \\
\hline $\begin{array}{l}\text { CC Sociales y } \\
\text { Jurídicas }\end{array}$ & 2 & 9 & 11 & 13 & $\begin{array}{l}\text { 1. Univ. Tiradentes; 2. Consejo Nal. de } \\
\text { Investigaciones Científicas y Técnicas / Univ. } \\
\text { de la Cuenca del Plata }\end{array}$ \\
\hline Ingenierías & 1 & 4.3 & 9 & 11 & Drexel University \\
\hline $\begin{array}{l}\text { Artes y } \\
\text { Humanidades }\end{array}$ & 1 & 4.3 & 2 & 2 & Universidade do Estado de Santa Catarina \\
\hline
\end{tabular}

Fuente: elaboración propia; en negrita aquellas instituciones, donde trabajan los IP, que se repiten

Hemos observado y contabilizado las filiaciones de los 23 investigadores principales. 17 de ellas, casi el $74 \%$ (17 investigadores) corresponden a universidades públicas o privadas, principalmente públicas, y 6 investigadores (26\%) trabajan únicamente en instituciones de docencia, investigación o gestión no universitarias. Debemos aclarar que 5 investigadores (21 
\%) cuentan con filiaciones que comparten tanto en universidades como en otras instancias. De las universidades (17 en total) pudimos extraer que 11 son de Brasil; a España y Argentina hay que adscribir 3 en cada país; de Colombia hemos de contabilizar 2 y las dos restantes se encuentran en Estados Unidos y México.

Por otro lado, de esta Tabla (4) también podemos extraer que la institución con el mayor número de frecuencias, 3 , (y por consiguiente destacada por su interdisciplinariedad) en el área de salud es la Fundação Oswaldo Cruz (Fiocruz), seguida, con 2 menciones, por la Escola Nacional de Saúde Pública Sérgio Arouca, al igual que el Consejo Nacional de Investigaciones Científicas y Tecnológicas. El resto de instituciones no se repite, lo que supone una dispersión en los resultados y da cuenta del nivel, bajo, de interdisciplinariedad.

Sobre los tres IP del campo de comunicación, destacaremos que se adscriben, respectivamente, a la Universidad Pompeu Fabra, Ministério da Saúde y al Centro Universitário Metodista Izabela Hendrix. Sin embargo, identificamos que 5 colaboradores de comunicación trabajan en la Universitat Autònoma de Barcelona. Ahora bien de fijarnos en los colaboradores del campo de la salud, 4 de ellos trabajan para la University of Texas at El Paso, 3 en el Instituto Nacional de Cáncer (Brasil), igual número en IOC/Fiocruz, y 2 se ubican en el Instituto Nacional de Salud Pública y en la Universidade de Brasília. A estos datos debe agregarse que en una ocasión una misma IP presentó dos filiaciones distintas (ENSPSA/Fiocruz; y Universidade Federal de Viçosa), y fue la única en repetir artículo con todo su equipo de colaboradores en una revista (Interface). Se trata de Kelly-Santos, Ribeiro y Monteiro; cuyos artículos se titularon "Acervo de materiais educativos sobre hanseníase: um dispositivo da memória e das práticas comunicativas" (2010) y "Hanseníase e práticas da comunicação: estudo de recepção de materiais educativos em um serviço de saúde no Rio de Janeiro" (2012).

Las cifras respecto a los colaboradores revelan que de los 85 en los 23 artículos, el $48 \%$ pertenecen al campo de la salud, porcentaje similar al caso de los IP; mientras que el $13 \%$ se inscriben tanto los investigadores de ciencias sociales y jurídicas como los de comunicación; un $11 \%$ son de ingenierías y $5 \%$ de ciencias de la naturaleza. En artes y humanidades solo se ubica un colaborador.

Ahora bien, si consideramos las relaciones interdisciplinarias con el campo de la salud (Gráfico 1), se aprecia que poco más de la tercera parte $35 \%$ (8 artículos) es con el de ciencias sociales y jurídicas, en tanto que con comunicación e ingenierías hay un $26 \%$ ( 6 artículos) y un 21\%, (5 artículos), respectivamente. Con artes y humanidades un $9 \%$ ( 2 artículos) y finalmente, se sitúa el campo de ciencias de la naturaleza, con un solo trabajo interdisciplinar con el campo de la salud. 


\section{Gráfico 1. Relaciones interdisciplinarias con el campo de la Salud.}

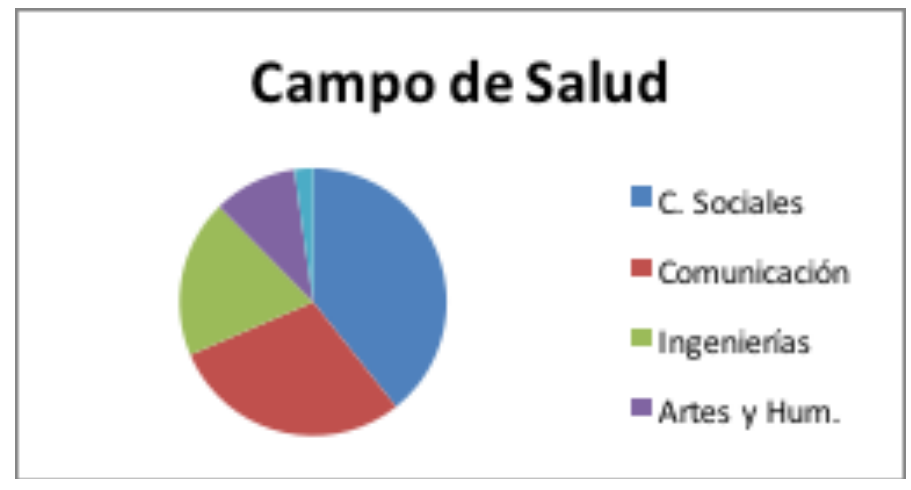

Fuente: elaboración propia

Por otro lado, si consideramos tanto al IP como a los colaboradores, deparamos que la salud constituye el área con más artículos (21), seguidos del de ciencias sociales y jurídicas (10), comunicación (7), Ingenierías (6) ciencias de la naturaleza (5); y artes y humanidades (1). Las relaciones entre las áreas de salud y comunicación se presentaron en el $26 \%$ de los artículos (6): Mas-Manchón et al (2015), "Valores percibidos en la publicidad de alimentos por jóvenes con y sin trastornos de la conducta alimentaria"; Kelly S-Santos et al. $(2010,2012)$, "Acervo de materiais educativos sobre hanseníase: um dispositivo da memória e das práticas comunicativas" y "Hanseníase e práticas da comunicação: estudo de recepção de materiais educativos em um serviço de saúde no Rio de Janeiro"; Vieira (2011), "Survey of rubella knowledge and acceptability of rubella vaccination among Brazilian adults prior to mass vaccination"; Grossman, Araújo-Jorge y Araujo (2008), "A escuta sensível: um estudo sobre o relacionamento entre pessoas e ambientes voltados para a saúde"; y Vives-Cases, Ruiz, Álvarez-Dardet y Martín (2005), "Historia reciente de la cobertura periodística de la violencia contra las mujeres en el contexto español (1997-2001)" -en este último artículo uno de sus colaboradores además pertenece al campo de las ciencias sociales-.

No obstante, hay dos artículos cuyos autores (tanto IP como colaboradores) no se adscriben al área de la salud. En el primer caso son investigadores de ciencias y ciencias sociales y jurídicas: Cubillas-Tejeda et al. (2011), "Diseño y aplicación de un Programa de Comunicación de Riesgos para la salud ambiental infantil en un sitio contaminado con plomo y arsénico"; y, en el segundo, corresponden a comunicación e ingenierías: Oliveira y Epstein (2009), "Ciência e consenso: os diferentes tempos que envolvem a pesquisa científica, a decisão política e a opinião pública". 
Tabla 5. Relaciones entre pares de campos de conocimiento por número de artículos

\begin{tabular}{|l|l|l|}
\hline Campo de Conocimiento & $\begin{array}{l}\text { Campos de Conocimiento con } \\
\text { que se relacionan }\end{array}$ & Número de artículos \\
\hline Salud & C. Sociales & 9 \\
\hline & Comunicación & 6 \\
\hline & Ingenierías & 4 \\
\hline & Ciencias & 4 \\
\hline & Artes & 1 \\
\hline C. Sociales & Ciencias & 3 \\
\hline & Ingenierías & 1 \\
\hline Comunicación & Ingenierías & 1 \\
\hline & C. Sociales & 1 \\
\hline
\end{tabular}

Fuente: elaboración propia

De la Tabla 5 se desprende que la mayoría de los artículos interdisciplinarios fueron entre el campo de la salud y el de las ciencias sociales, comunicación incluida (15 artículos); con los otros campos la interrelación desciende claramente. Como puede apreciarse, las conexiones disciplinarias más frecuentes en esta muestra son la del campo de la salud con los 5 campos restantes considerados. En las demás relaciones disciplinarias identificamos poca interrelación, incluso en comunicación, con 3 combinaciones: salud, ingenierías y ciencias sociales y jurídicas. Debemos advertir que la Tabla 5 muestra la interdisciplinariedad de manera binaria, incluyendo todas las posibles combinaciones, por lo que la suma es superior a 23.

Tres artículos interrelacionan tres campos de conocimiento. En 2 de ellos su IP es de ciencias, mientras que sus colaboradores son de ciencias sociales y jurídicas y de salud: Sanmartino et al. (2015), "Que no tengan miedo de nosotros: el Chagas según los propios protagonistas" y Passamai et al (2012), "Letramento funcional em saúde: reflexões e conceitos sobre seu impacto na interação entre usuários, profissionais e sistema de saúde". El otro artículo, con el IP en el área de la salud e interrelaciones con ciencias sociales y jurídicas y con ingeniería, es el de Leonardo Gómez y Piacenza (2010), "Homicidios diarios. Análisis del discurso periodístico sobre homicidios por armas de fuego. Buenos Aires (Argentina) 2001-2002".

Tabla 6. Artículos con equipos interdisicplinarios por revista y número de campos (organizado alfabéticamente y cronológicamente)

\begin{tabular}{|l|l|l|l|l|}
\hline Revista & Año & IP & $\begin{array}{l}\text { Núm. De } \\
\text { Cols. }\end{array}$ & $\begin{array}{l}\text { Núm. de } \\
\text { Campos }\end{array}$ \\
\hline Cadernos Saude Publica & 2014 & Landini, F. & 2 & 2 \\
\hline Ciênc. saúde coletiva & 2011 & Cubillas-Tejeda, A & 7 & 2 \\
\hline Gaceta Sanitaria & 2013 & Leis, Á. & 5 & 2 \\
\hline
\end{tabular}




\begin{tabular}{|c|c|c|c|c|}
\hline Interface (Botucatu) & 2015 & Sanmartino, M. & 4 & 3 \\
\hline$"$ & 2014 & de Aguiar, M. & 1 & 2 \\
\hline$" 1 "$ & " " & de Longhi, $A$. & 3 & 2 \\
\hline$"$ & 2012 & Cavaca, A. & 3 & 2 \\
\hline$"$ & " " & Kelly-Santos, A. & 2 & 2 \\
\hline$"$ & $" 1$ & Passamai, M. & 3 & 3 \\
\hline$"$ & 2011 & Paixão, P. & 5 & 2 \\
\hline$"$ & 2010 & Kelly-Santos, A. & 2 & 2 \\
\hline$"$ & 2009 & Oliveira, J. & 1 & 2 \\
\hline$"$ & $" 1 "$ & Oliveira, S. & 2 & 2 \\
\hline$"$ & 2008 & Grossman, E. & 2 & 2 \\
\hline$"$ & 2005 & Vives-Cases, C. & & \\
\hline Rev. Panam Salud Publica & 2011 & Vieira, J. & 4 & 2 \\
\hline$" 1 "$ & 2009 & Galada, $\mathrm{H}$. & 6 & 2 \\
\hline $\begin{array}{l}\text { Revista Peruana de } \\
\text { Medicina Experimental y } \\
\text { Salud Pública }\end{array}$ & 2015 & Berrospi, V. & 5 & 2 \\
\hline Revista Salud Pública & 2012 & Flórez-Romero, R. & 2 & 2 \\
\hline " $" 1$ & 2006 & Rodriguez, J. & 3 & 2 \\
\hline Salud Colectiva & 2015 & Mas-Manchón, L & 8 & 2 \\
\hline " " & 2010 & Federico, L. & 2 & 3 \\
\hline Salud Pública de México & " " & Thrasher, J. & 10 & 2 \\
\hline
\end{tabular}

Fuente: elaboración propia

De la Tabla 6 se desprende que Interface (con dos) y Salud Colectiva (con 1) son las únicas revistas que han publicado artículos con autores de 3 campos de conocimiento. En cuanto al número de coautores, encontramos un máximo de 10 (lo cual sería imposible en el campo de las ciencias sociales y jurídicas) en un caso y otro artículo con un solo coautor. Lo más habitual es la coautoría de 3 , incluido el IP. No obstante, el número de coautores, si atendemos a los 23 artículos, es superior al que se puede encontrar en la mayoría de los artículos del campo de la comunicación.

En cuanto a la tipología de los artículos, recordemos que si la muestra es la de los 124 que tratan sobre comunicación (Terrón, Ramírez, Vialás y Jacobetty, 2016) el resultado es el que sigue: 115 'artículos originales', 6 'revisiones', 1 como 'reseña bibliográfica', 1 en calidad de 'comentario' y 1 como 'perspectivas.' Ningún escrito entraba en la clasificación de 'carta al director' o de 'editorial.' Los escritos que ahora revisamos (23) en su mayoría, 19, son artículos originales.

De fijarnos en los enfoques metodológicos, obtendremos los siguientes resultados: 
Tabla 7. Artículos empíricos

\begin{tabular}{|l|l|l|}
\hline Empíricos & Sobre 124 & Sobre 23 \\
\hline Cuantitativos & $43(34,67 \%)$ & $10(43,47 \%)$ \\
\hline Cualitativos & $31(25 \%)$ & $8(34,78 \%)$ \\
\hline Mixtos & $4(3,22 \%)$ & $1(4,34 \%)$ \\
\hline Total & $78(62,9 \%)$ & $19(82,6 \%)$ \\
\hline
\end{tabular}

Fuente: elaboración propia.

Vemos que las proporciones no varían de manera ostensible entre las dos muestras (la mayor desviación la encontramos en el total de artículos empíricos, superior entre los escritos interdisciplinarios), en las que prevalecen las investigaciones cuantitativas, aunque con un número de considerable de investigaciones cualitativas.

En cuanto al resto de posibilidades, tipos de investigación, destacar que 3 son teóricas conceptuales (21 sobre 124) y 1 metodológica (5 sobre 124); no hay ninguna revisión (6 sobre 124). Por el contrario, ninguno de los 23 artículos está exento de explicitar sus técnicas y enfoques metodológicos (lo que si ocurría en 14 sobre 124 artículos).

En relación a los enfoques, en estos 23 artículos prevalecen las encuestas (en 6 ocasiones), seguidos de los análisis de contenido (en 3), estudios de caso (en 3), análisis del discurso (en 2), los cuestionarios (en 2) y las entrevistas (en 2). En 1 caso nos encontramos con el análisis secundario de datos y el focus grup.

Si tenemos en cuenta los contenidos, y tras la agrupación en pro de una mayor legibilidad que explicamos en el apartado de metodología, los resultados son los siguientes: destacan aquellos centrados en la alfabetización de la salud (10 artículos), seguidos ya a cierta distancia de los que se centran en las desigualdades $(3$, y con una correlación muy clara con los artículos sobre alfabetización de la salud), el medio ambiente (3), los riesgos y la violencia (3). Dos artículos hemos de consignar dentro de las categorías de publicidad y de tecnología. Y con 1, la comunicación médico paciente y la divulgación científica. No deja de sorprendernos el número tan bajo de artículos centrados en la tecnología teniendo en cuenta la importancia creciente de las TIC en la práctica sanitaria. Por contra, podemos afirmar que en mayor o menor medida todos los artículos (23) se marcan como objetivo la promoción de la salud.

Si atendemos a qué asuntos relacionados con la salud y las patologías tratan los artículos analizados, observamos que son muchos menos de que los que nos pudiéramos pensar en un principio: trastornos alimentarios ( 2 artículos) y en 1 artículo se trata sobre el mal de chagas, la lepra, el monóxido de carbono, el tabaquismo y la salud bucal. Hemos de subrayar que no hay ninguno que se centre en la salud mental.

Y en relación a los medios de comunicación, reseñar que estos aparecen de manera explícita en 9 artículos: en 3 ocasiones los diarios y en 1 ocasión la cobertura periodística (la agenda informativa), Facebook, internet, los medios de comunicación social, el periodismo científico y la televisión. 


\section{Discusión}

Antes de desarrollar este apartado, nos parece pertinente señalar algunas limitaciones que imposibilitan que los resultados obtenidos puedan generalizarse; son, por tanto, el resultado de una muestra, sita en un contexto, que nos puede hablar del mismo, pero no de todo el mundo. Tengamos en cuenta que la investigación se ciñe al ámbito latinoamericano; quizás, por ejemplo, en el anglosajón, donde la comunicación y salud está más desarrollada, las proporciones difieran; por tanto, se precisan estudios similares que analicen lo que pasa en otras áreas geográficas. Por otro lado, hemos puesto nuestro foco de atención sobre las revistas de salud pública porque pensamos que serían las más propensas a centrarse en el campo de la comunicación y quizás esta sea una apreciación errónea, puede que en el campo de la salud en su conjunto, o atendiendo a algunas especialidades, el tanto por ciento de originales dedicados a la comunicación sea mayor, pero sólo tras hacer las investigaciones pertinentes podremos llegar algún tipo de conclusión.

Eso sí, tras nuestra investigación estamos en condiciones de asegurar que la comunicación es algo meramente instrumental para la salud pública. Recordemos que 6 de las 18 revistas estudiadas no insertan ningún artículo que se centrara en la comunicación y que sólo el 0,62\% de los artículos (124 sobre 19.886) analizados giraban en torno a la comunicación. A la vez, vemos que con el paso de los años no se va incrementando el número de originales que traten sobre comunicación, que los 124 artículos se concentran en sólo 88 ejemplares, que tan sólo dos publicaciones dedican un dossier a la comunicación y que entre las 124 piezas no hay ningún editorial y salvo en una revista, Interface, en ninguna otra aparecen artículos sobre comunicación en los 11 años estudiados, revista, por cierto, que supone el 41,12\% de los artículos analizados. Podemos hablar, por tanto, de una cierta concentración de escritos, conclusión que también avala el hecho de que si deparamos en las 5 revistas que insertan 10 o más escritos, identificamos que estos suman el $78,22 \%$ del total.

De prestar atención a los artículos interdisciplinares, observamos que estos (23) suponen apenas el 18,5\% de los artículos que hablan de comunicación (124) y un 0,63\% del total de los artículos (19.886) que publican las 12 revistas estudiadas. Resultados que ponen de relieve la poca incidencia, durante el periodo analizado, de los trabajos interdisciplinarios de las revistas de SP que se incluyen en SCIELO. Esta poca presencia de artículos con autores de diversas disciplinas se opone al perfil de la SP, la cual, para Nunes $(1994,19-20)$ se fundamenta "en una interdisciplinariedad, como posibilitadora de construcción de un conocimiento ampliado de salud, y una multiprofesionalidad, como forma de enfrentar una diversidad interna del saber/ hacer de las prácticas sanitarias", en línea con lo que mantiene la propia OPS (2012).

Eso sí, la interdisciplinariedad, aunque escasa en su conjunto y entre campos de conocimiento, es mayor entre los campos de la salud y de las ciencias sociales y de las humanidades, lo cual, en cierta forma, responde a lo que Nunes $(1994,20)$ refiere como vocación de "la salud colectiva -constituida en los límites de lo biológico y lo social-", orientada a fomentar el análisis, entendimiento e interpretación de los determinantes de la producción social de la enfermedad y organización social de los servicios de salud, en distintos momentos y trayectorias históricas.

Desde nuestro punto de vista, la diversidad de contextos socioculturales de las relaciones entre producción de conocimientos en salud, las formas y procesos de transmisión y la 
correspondiente apropiación de distintos grupos humanos, señaladas por Castiel (2003), no parece reflejarse, en estas publicaciones, de la multiplicidad de relaciones entre disciplinas científicas que dan cuenta de las mismas. Aun cuando nadie objetaría la riqueza analítica producida por la diversidad de enfoques y el diálogo de especialistas de distintas formaciones, en la práctica es mínima la producción disponible que "conjugue conceptos e intereses disímiles y cruce diversos lenguajes e impulsos disciplinarios. Asimismo, es preciso rescatar el sustrato normativo que opera como ancla de la investigación, vale decir, las premisas y objetivos políticos que motivan y subyacen al trabajo académico" (Petracci y Waisbord, 2011:12). Comotampoco se podrá argumentar que estas cifras representen el incremento de un esfuerzo encaminado a enfrentar las diferencias de los respectivos lenguajes de quienes participan (como productores, transmisores y receptores) en estos procesos (Castiel, 2003, 164).

Esta baja interdisciplinariedad lógicamente también se da entre los campos de la salud y de la comunicación. Por un lado, nos encontramos con autores que ven una estrecha relación entre comunicación y salud pública (Algarra; 1997; Gehner, 1999; de Lorenzo, 2004; Cuesta; Gaspar, 2013; Hernán Díaz, 2014; Revuelta, Faubel y Carrasco, 2014); de hecho, Martínez Pecino, Reig y Marín Sánchez (2013), tras una revisión de 10 años de artículos de revistas científicas españolas del ámbito de la comunicación, nos dicen que la mayor parte de los artículos que giran en torno a la salud, tratan de salud pública (un 1\% del total de artículos, 1962 referencias). Pero, por otro, como hemos señalado (OPS, 2012, Barragán, 2007), la comunicación aún no ha acabado de integrarse en los estudios curriculares de la salud pública ni es entendida como una de las ciencias con las que debe imbricarse en la misma.

La interdisciplinariedad entre los campos de la salud y los de las ciencias de la naturaleza y de las ingenierías es aún menor, lo cual no deja de sorprendernos, en este segunda caso, dado el rol, cada vez más preponderante, de las tecnologías de la información y de la comunicación en todos los procesos de que atañen a la salud pública y a la medicina.

La baja interdisciplinariedad de la que venimos hablando se traduce en una reducida respuesta por parte de los investigadores de estas disciplinas para buscar explicaciones y nuevos planteamientos sobre cuál es la identidad salud pública, que en palabras de Campos (2000) consistiría en cuestionarse sobre ¿cuál es su núcleo conocimientos y prácticas?, ¿en qué campo de interinfluencia que estaría inmerso?, y ¿quiénes son los agentes que propician la salud pública?

En el apartado de resultados mostramos como, generalmente, las relaciones entre los campos de conocimiento son binarias. Así, el de la salud privilegia las ciencias sociales, alcanzando un $34 \%$, le siguen la comunicación, con menos de la cuarta parte (que no hemos de olvidar que forma parte de las ciencias sociales), y después las ingenierías y las ciencias de la naturaleza, con $17 \%$ cada una. Si bien es cierto que no siempre es necesaria la interdisciplinariedad (e incluso su obligatoriedad podría ser ridícula), parecería deseable que esta fuera mayor, tal como manifiesta de Souza (2008), quien agrega que ninguna disciplina sola se basta para explicar y comprender la realidad compleja de la salud, por lo cual convoca a la apertura a nuevos abordajes teóricos y metodológicos. "No hay dudas de que cada disciplina genera conocimientos propios, los cuales son importantes tanto para el avance de la ciencia como para iluminar determinados aspectos de la realidad. Pero me gustaría revalorizar el diálogo, el 
intercambio y la combinación entre diversos saberes." (de Souza, 2008:7).

Abundando en lo que venimos escribiendo, que ésta, la interdisciplinariedad cuando se produce se limita en la gran mayoría de los casos a la connivencia entre dos campos, reduce el potencial de la misma. Pensamos que este fenómeno es el resultado no sólo del desconocimiento o de la poca valoración de otros campos, sino también de la complejidad administrativa que conlleva el trabajar con personas de otros entes investigadores (sean instancias laborales o campos).

Si prestamos atención a las revistas, vemos que una sola publicación, Interface, contiene casi el $50 \%$ de los artículos interdisciplinares; de nuevo nos encontramos, en primer lugar, ante una concentración de resultados (debemos añadir que otra tres revistas - Revista de Salud Pública, Salud Colectiva y Revista Panamericana de Salud Pública-, con dos artículos cada una, suman otra cuarta parte del total) y, en segundo término, ante el hecho excepcional de la revista Interface, que, en sí misma, contradice los resultados generales que hemos obtenido. Por otro lado, Interface (con dos) y Salud Colectiva (con uno) son las únicas revistas que han publicado artículos con autores de 3 campos de conocimiento. De nuevo podemos hablar de 'concentración'; por tanto, que la interdisciplinariedad no está generalizada.

Siguiendo con este apartado, dado los resultados a los que llegamos y que estos responden a cantidades poco significativas, no podemos establecer tendencia alguna en las relaciones entre IP, colaboradores o centros de trabajo. Así como podemos considerar como anecdóticos, en relación a los resultados y al discurso interdisciplinar, los equipos estables de investigadores de diferentes campos del conocimiento.

No obstante, y seguramente por tratarse de revistas de salud, el $52 \%$ de los IP son de este campo, al que le siguen el $21 \%$ de ciencias sociales y jurídicas (agregando comunicación), el $17 \%$ de ciencias de la naturaleza y el $4,3 \%$ a ingenierías y artes y humanidades. Aunque las proporciones varíen ligeramente, el mismo orden se da en la muestra de 124 artículos y ocurre de igual forma entre los colaboradores de los 23 escritos interdisciplinares. Estos resultados, en primer lugar, deben hacernos reflexionar sobre el interés o las barreras de las publicaciones de un campo dado en admitir originales de IP de otros campos. En este caso, la falta de interdisciplinariedad sería propiciada por la propia revista y se trataría, por así decirlo, de una ausencia estructural. $Y$, en segundo lugar, como un campo en concreto atrae un mayor número de IP y colaboradores de ese mismo campo aún cuando hay interdisciplinariedad.

En cuanto a las filiaciones de los IP, tengamos presente que 17 pertenecían a universidades (principalmente públicas) y 6 a otras instituciones de investigación ( 5 tenían una doble filiación). Resultados que concuerdan con la realidad de unos países en los que la investigación se concentra en las universidades.

En cuanto a la tipología de los artículos, como ya hemos demostrado (Terrón, Ramírez, Vialás y Jacobetty, 2017), nuestros resultados contradicen a los de Bellón y Martínez Cañabate (2001), quienes concluyen que hay un exceso de revisiones y una falta de artículos originales. Si tomamos como referencia los 124 artículos que tratan sobre comunicación, 114 son originales y 6 revisiones; en el caso de los 23 interdisciplinares, 19 son artículos originales. Recordemos que en la investigación de Bellón y Martínez Cañabate (2001), la proporción de artículos originales 
en MEDLINE e IME fue, respectivamente, $70 \%$ y $37 \%$, y de revisión $11 \%$ y $44 \%$. Estos resultados tan dispares nos obligan a preguntarnos por qué se producen y deberían traducirse en nuevas investigaciones que den respuesta a esta pregunta.

Y en relación a los enfoques metodológicos, como ya hemos explicado (Terrón, Ramírez, Vialás y Jacobetty, 2017), no disponemos de investigaciones similares con las que comparar nuestro estudio, pero, siendo conscientes de sus limitaciones, leeremos nuestros resultados con la ayuda de Martínez Nicolás y Saperas (2016), Thomson, T.L. (2006) y Freimuth, V.S. et al (2006).

Tanto si tomamos como referencia los 124 artículos como los 23 interdisciplinares, en las muestras que estudiamos se da un mayor equilibrio entre técnica cuantitativas y cualitativas de las que existe en el campo de la comunicación; lo mismo sucede entre artículos empíricos y no empíricos, cuando cabría pensar que por ser revistas de salud los resultados podrían ser los contrarios. Mantenemos que esto es así, en gran medida, debido a la tradición académica e investigadora de la salud pública.

Tenemos que añadir que en nuestras muestras la cantidad de artículos teórico-conceptuales y metodológicos es sustancialmente superior al de las muestras con las que los comparamos; pero eso sí, casi la mayoría los inserta Interface. $Y$ hemos de destacar que ninguno de los 23 artículos originales están exentos de explicitar sus técnicas y enfoques metodológicos (lo que si ocurría en el $12 \%$ de los 124 artículos o en el $20 \%$ de la muestra de Martínez Nicolás y Saperas), de lo que deberíamos deducir que la interdisciplinariedad favorece la calidad de los originales.

Por último, y en relación a los contenidos, debemos resaltar que los 23 artículos tienen como objetivo la promoción de la salud, una de las finalidades de la salud pública (OMS, 2012); además, y en esta misma línea, cabe añadir que 10 artículos se centran en la alfabetización de la salud y, como ya escribimos en el apartado de resultados, 3 deparan en las desigualdades (con una correlación muy clara con la alfabetización sobre salud y con los que tratan sobre la violencia), 3 en riesgos y violencia y 3 en el medio ambiente. Como se puede apreciar, todos ellos son determinantes sociales, uno de los pilares que sostiene la salud pública contemporánea. A dos artículos los hemos de consignar dentro de las categorías de publicidad y de tecnología. Y a 1, en la comunicación médico paciente y la divulgación científica. Ya hemos apuntado que no deja de sorprendernos el número tan bajo de artículos centrados en la tecnología, si deparamos en la importancia creciente de las TIC en la práctica sanitaria. También nos sorprende que un solo artículo aborde la relación médico-paciente, que puede y debe ser vista en muchos casos desde una perspectiva interdisciplinar (por ejemplo, la tecnológica, la de la comunicación y la de la salud).

En cuanto a qué asuntos relacionados con la salud y las patologías tratan los artículos analizados más de lo que se trata es importante considerar de qué no se habla: la salud mental, lo cual tampoco es nada sorprendente si atendemos a su constante minusvaloración dentro de los planes de salud, lo que se debe, en parte, a la preeminencia del modelo biomédico sobre el biopsicosocial. 


\section{Conclusiones}

Párrafos atrás mencionamos las limitaciones de esta investigación: hablamos de revistas de salud pública en un contexto sociocultural concreto, el latinoamericano, por lo que los resultados que expondremos no deben extrapolarse ni a otros contextos geográficos (por ejemplo, el anglosajón) ni al conjunto de las revistas del campo de salud. Como hemos escrito, otras investigaciones deberán decirnos si los resultados que hemos obtenido son propios de las revistas de salud pública latinoamericanas o se asemejan a los que las revistas de salud pública de distintas latitudes.

No obstante, consideramos que de nuestro estudio se pueden extraer algunas conclusiones que merecerían reflexiones pausadas y que deberían, desde nuestro punto de vista, ser una llamada de atención para los campos de la salud (y más específicamente de la salud pública) y de la comunicación.

La primera conclusión a la que podemos llegar, y de esta forma ir dando respuesta a las preguntas que nos hacíamos en el apartado metodológico, es que para la salud pública la comunicación, en la mayoría de los casos, es algo meramente instrumental. De ahí ese escaso 0,62\% de artículos (124) que según la propia SCIELO están dedicados a la comunicación; o que en 6 de las 18 revistas de la muestra no se publique ninguno. Sin olvidarnos que los artículos examinados se concentran en 88 números y que una revista, Interface, reúne el $41,12 \%$ de los artículos de esta muestra, convirtiéndose en un caso, en una publicación singular.

Si deparamos en la cantidad de artículos interdisciplinares, vemos que estamos hablando de 23 , o sea, el $18,5 \%$ de los artículos que hablan de comunicación (124) y un 0,11\% del total de los artículos (19.886) que publican las 12 revistas estudiadas. Es evidente que en el caso que nos ocupa la interdisciplinariedad es baja y que los resultados contradicen las directrices que han de guiar a la salud pública. Por otro lado, el campo de la salud, lo cual tiene su lógica dado que hablamos de revistas de salud, y los IP de este campo vertebran mayoritariamente esa interdisciplinariedad, en la que la comunicación ocupa un segundo lugar en esa interrelación entre campos, tras el conjunto de saberes que conforman las ciencias sociales y jurídicas. Lo escrito entronca, como hemos citado, con la importancia que le da la salud pública a las ciencias sociales, aunque cuando habla de estas no incluye a las ciencias de la comunicación.

Esa baja presencia de la comunicación nos habla de que el campo de la comunicación y salud es aún más un deseo que una realidad, o como venimos diciendo, que para la salud la comunicación es un mero instrumento para sus finalidades, sin que exista un reconocimiento que si se concede a otras ciencias sociales (demografía, sociología, etnografía, ciencias políticas, etc.). Por otro lado, esa baja interdisciplinariedad se pone de manifiesto en que, fuera de algún indicio, no parecen existir equipos estableces interdisciplinarios, ni una relación entre instituciones investigadoras que vayan más allá de los episódico y salvo en tres artículos (en los que aparecen tres campos) lo usual es una interdisciplinariedad binaria. Como tenemos que consignar que una revista, Interface, publica 11 de esos 23 artículos, por lo que, de nuevo, tenemos que escribir de caso singular y de concentración.

Otra manera de enfocar el tema de la interdisciplinariedad es fijarnos en la tipología de los escritos, recordemos, 19 artículos originales, 3 teórico conceptuales y 1 metodológico. Los 
resultados, proporcionalmente, son semejantes a los que obtuvimos de fijarnos en los 124 artículos, pero en este caso debemos subrayar que estamos hablando de artículos en los que se teoriza de manera interdisciplinaria, lo cual les concede un mayor valor, aunque sólo supongan un $18,18 \%$ de la muestra de los escritos interdisciplinarios. Por otro lado, hemos de remarcar que ninguno de los 23 artículos está exento de explicitar sus técnicas y enfoques metodológicos (lo que si ocurría en 14 sobre 124 artículos), lo que nos lleva a concluir que bajo ese parámetro, muy importante, los artículos interdisciplinares tienen una mayor calidad.

En cuanto a los contenidos, cabe remarcar que los 23 artículos buscan promocionar la salud, una de las finalidades de la salud pública, y en la mayoría de ellos, a la vez, empoderar al destinatario.

Por último, consideramos importante leer los resultados obtenidos desde otra perspectiva, el de las revistas. ¿Hasta qué puntos propician las publicaciones los trabajos interdisciplinares? ¿Qué dificultades añadidas se dan para publicar en una revista de un campo diferente al del IP? Sabemos que las trabas administrativas perjudican la interdisciplinariedad, dado quela dificultan; de alguna manera, ¿las publicaciones de un campo, en este caso de la salud, restringen la participación de autores de otros campos aún en el supuesto de poder hacer aportaciones al mismo? Creemos que esta es una pregunta capital a la que debemos encontrar respuestas en próximas investigaciones para, dependiendo de las mismas, pedir a los editores de las revistas que no sólo defiendan la interdisciplinariedad, sino que la promuevan atendiendo a unas prácticas que habrá que definir.

\section{REFERENCIAS BIBLIOGRÁFICAS}

- Algarra, M. (1997). Las campañas de comunicación pública. La comunicación y salud como campo de estudio. Communication \& Society 10(1), 193-201.

- $\quad$ Almeida Filho, N. \& Silva Paim, J. (1999). La crisis de la salud pública y el movimiento de la salud colectiva en Latinoamérica. Cuadernos médico sociales, 75, 5-30.

- Barragán, H.L. (2007). Fundamentos de salud pública. La Plata: Editorial de la Universidad Nacional de la Plata.

- $\quad$ Baum, F. (2007). The new public health. Oxford: Oxford University Press.

- Bellón, J.A. \& Martínez Cañabete, T. (2001). La investigación en comunicación y salud. Una perspectiva nacional e intenacional desde el análisis bibliométrico. Atención Primaria, 27 (7): 452-458. DOI: 10.1016/S0212-6567(01)78835-X.

- Campos, G. W. (2000). Saúde pública e saúde coletiva: campo e núcleo de saberes e práticas. Ciência \& Saúde Coletiva, 5(2), 219-230. DOI: 10.1590/S141381232000000200002

- Castellanos, P. (1998). Los modelos explicativos del proceso salud enfermedad: los determinantes sociales, 81-102. En Martínez, F, Castellanos, P. L. \& Navarro, V. (Coords.) Salud Pública. Ciudad de México: Mc Graw-Hill.

- Castiel, L. (2003) Insegurança, ética e comunicação em saúde pública. Rev Saúde Pública. 37(2),161-167.

- Conde, F. \& Pérez, C. (1995). La investigación cualitativa en salud pública. Rev. Esp. Salud Pública, 69 (2), 145-149.

- Cuesta, U., \& Gaspar, S. (2013). Publicidad y salud. Madrid: Fragua.

- Díaz, Hernán (2014). El papel de la comunicación en la salud, 4-5. Comunicant salut, 
Valencia: Consellería de Sanitat. Generalitat.

- $\quad$ Ferreira Neto, J. L.. Kind, L., Pereira, A. Barbosa, Rezende, M. C., \& Fernandes, M. L. (2011). Usos da noção de subjetividade no campo da Saúde Coletiva. Cadernos de Saúde Pública, 27(5), 831-842. https://dx.doi.org/10.1590/S0102-311X2011000500002

- Franco, A. (2006). Tendencias y teorías en salud pública.Rev. Fac. Nac. Salud Pública, 24(2)- 119-130.

- $\quad$ Freimuth, V.S. et al (2006). A Descriptive Analysis of 10 Years of Research Published in the Journal of Health Communication, Journal of Healt Communication, 10 (1), 11-21. DOI: 10.1080/10810730500461042.

- Frenk, J. (1992). La nueva salud pública, 75-93. En OPS, La crisis de la salud pública, reflexiones para el debate, Pub. Científicas, 540.

- Gehner, M. (1999). Comunicación para una mejor salud global, Quark, 16. Consultado el día 2 de septiembre de 2016 de la World Wide Web: http://quark.prbb.org/16/

- Kottow, M. (2013) Pierre Bourdieu: sociología reflexiva para salud pública y su bioética. Nuevos folios de bioética, 12, 5-21.

- de Lorenzo, R. (2004). Salud y comunicación, CONAMED, 9(4), 26-33.

- Lois, I. (2015). Notas sobre las perspectivas, límites y desafíos de la comunicación y salud. En Kornblit, A., Camarotti, A. \& Güelman, M. X Jornadas Nacionales de Debate Interdisciplinario en Salud y Población, Buenos Aires. URL: https://www.teseopress. com/jornadasnacionalessalud

- Martínez Nicolás, M., \& Saperas-Lapiedra, E. (2016): Objetos de estudio y orientación metodológica de la reciente investigación sobre comunicación en España (2008-2014). Revista Latina de Comunicación Social, 71 1365- 1384. DOI: 10.4185/RLCS-2016-1150es.

- Martínez Nicolás, M., \& Saperas Lapiedra, E. (2011): La investigación sobre Comunicación en España (1998-2007). Análisis de los artículos publicados en revistas científicas. Revista Latina de Comunicación Social, 66, 101-129. DOI: 10.4185/ RLCS-66-2011-926-101-129.

- Martínez Pecino, R., Reig, R., \& Marín Sánchez, M. (2013). Una década del mensaje sobre la salud en las revistas de comunicación indexadas en IN-RECS, Mensaje Periodístico, 19(2), 1047-1059.

- $\quad$ Navarro, V. (1998). Concepto actual de salud pública. En Martínez, F.; OPS (2012). La salud pública en las Américas. Washington: OPS. Publicación científica y técnica n 589.

- Petracci, M., \& Waisbord, S. (Comps.) (2011). Comunicación y salud en la Argentina. Buenos Aires: La Crujía.

- Ramos Domínguez, B.N. (2000). La nueva salud pública. Revista Cubana de Salud Pública, 26(2). Consultado el día 3 de febrero de 2016 de la World Wide Web: http:// scielo.sld.cu/scielo.php?script=sci arttext\&pid=S0864-34662000000200001

- Revuelta, G., Faubel, R., \& Carrasco, J.M. (2014): "Comunicació i salut pública", 8-9. Comunicant salut, Valencia: Consellería de Sanitat. Generalitat.

- de Souza. Interdisciplinariedad y pensamiento complejo en el área de la salud. Salud colectiva, Buenos Aires, 4(1):5-8.

- Scliar, M. (2002). Saúde, cultura e democracia. Saúde e Sociedade 11(1), 117-123.

- Thompson, T.L. (2006). Seventy-Five (Count'Em-75). Issues of Health Communication: An Analysis of Emerging Themes, Health Communication, 20(2), 117-123. DOI: 10.1207/ s15327027hc2002_2.

- Terrón, J. L., Ramírez, F.; Vialás, S. \& Jacobetty, P. (2016), "Los contenidos sobre 
comunicación en las revistas de salud pública. El caso de SCIELO", 613-642, en Herrero, J.; Mateos, C. (coords.) (2016). Del verbo al bit. La Laguna: Cuadernos Artesanos de Comunicación. ISBN: 978-84-16458-41-7

- Terrón, J. L., Ramírez, F., Vialás, S. \& Jacobetty, P. (2017). La comunicaciónen las revistas de salud pública. El caso SCIELO. Revista española de Comunicación en Salud, Suplemento 2, 27-28.

- Testa, M. (1992). Salud pública: acerca de su sentido y significado, 205-220. En: OPS, editor. La crisis de la salud pública: reflexiones para el debate. Washington: OPS.

- Vega Franco, L. (2000). La salud en el contexto de la nueva salud pública, Salud Pública de México, 42(4), 370-372.

\section{CURRICULUM VITAE}

\section{José Luis Terrón Blanco}

Doctor en Ciencias de la Comunicación por la Universidad Autònoma de Barcelona (UAB). Profesor Titular en el Departament de Comunicació Audiovisual i Publicitat de la UAB. Ha sido Director del InCom-UAB y ha fundado y dirige el Grupo de Comunicación y Salud del InComUAB. Especializado en comunicación y salud. Forma parte del comité científico de revistas científicas y del Informe de la Comunicació en Cataluya y del Observatori de la Comunicació en Catalunya (OCC). Dirige InCom-UAB Publicaciones.

\section{Flor Micaela Ramírez Leyva}

Profesora Titular del Departamento de Psicología del Centro Universitario de la Costa, de la Universidad de Guadalajara. Es miembro del Comité de Bioética en Investigación y colaboradora del Cuerpo Académico de Análisis Regional y Turismo del CuCosta, y del SGR de Comunicación del Incom-UAB. Maestría en Sociología de la Cultura, por la Universidad Autónoma de Aguascalientes, Doctora por la Universidad Miguel Hernández. Forma parte del comité editorial de revistas científicas de España, Chile y México. Sus líneas de investigación son comunicación y salud y medios de comunicación, género y poder, cuenta con varias publicaciones en estas áreas.

\section{Simón Vialás Fernández}

Licenciado en Comunicación Audiovisual en la Universidad Carlos III de Madrid. Doctor en Comunicación por la Universitat Autònoma de Barcelona. Su tesis Producción orientada al procomún en el documental español ha recibido la mención cum laude. Es investigador en el Instituto de la Comunicación (InCom-UAB) y sus líneas de investigación son tecnopolítica, comunicación para el cambio y comunicación y salud. Coordina cursos de especialización sobre TIC en la UAB.

\section{Pedro Jacobetty}

Licenciado en Sociología por el Instituto Universitario de (IUL) y doctor en Sociedad de la Información y el Conocimiento por la Universitat Oberta de Catalunya (UOC). Ha colaborado como asistente y consultor en varios proyectos de investigación del Centro Investigãçao e estudos em Sociologia (CIES-IUL), del Internet Interdiscuplinary Institue (IN3-UOC) y del grupos EduL@b. Sus intereses de investigación están conectados con la cultura digital, la comunicación, losestudios sociales de la ciencia, y la sociología de la educación. 
